\title{
ELECTRICAL CHARACTERIZATION OF ATMOSPHERIC PRESSURE GASEOUS DISCHARGE
}

\author{
S. R. Kandel, D. P. Subedi* \\ Department of natural Sciences, School of Science, Kathmandu University \\ Dhulikhel, Kavre, Nepal \\ Corresponding author: deepaksubedi2001@yahoo.com \\ Received 1 August; Revised 13 September
}

\begin{abstract}
This paper reports the generation of atmospheric pressure gaseous discharge and its electrical characterization. For this purpose, a point-plane electrode system has been fabricated. A high voltage power supply $(0-11 \mathrm{kV}, 50 \mathrm{~Hz})$ was applied across the electrodes. Discharge was generated with and without the dielectric barrier between the electrodes. The gas discharge was seen as in-homogenous filamentary type discharge without the dielectric. Whereas the filamentary discharge turned into bluish glow when the lower electrode was covered by a dielectric. The current and voltage of the discharge were measured by using a high frequency digital oscilloscope. The power consumed by the discharge was measured from the measured values of current and voltage. A special attention has been paid to investigate the effect of electrode geometry on the nature of the discharge
\end{abstract}

Key words: Gaseous discharge, point-plane electrode, dielectric material, filamentary discharge, current and voltage measurement

\section{INTRODUCTION}

Recently, much attention has been paid to the development of atmospheric pressure nonthermal plasma sources. The dielectric barrier discharge (DBD), as one of the main nonequilibrium plasma sources, can generate a non-equilibrium plasma under atmospheric pressure, requiring no expensive vacuum equipment, which is a must for conventional low-pressure glow discharge, and so processing cost is reduced and the ability of continuous processing is improved, and therefore large-scale industrial applications are possible [5-7]. Because of the thermal non-equilibrium between electrons and the bulk of the plasma, many physico-chemical reactions of interest can be carried out at near ambient temperature without any damage to the bulk of the material.

The term gas discharge is used to describe the flow of electric current through gases. The requirements for such passage of current are that some of the gas particles should be ionized and that there should exist an electric field to drive the charged particle so produced to form a current [1]. This clearly indicates that the gas must be ionized, completely or partially, either by supplying heat or by supplying high voltage across the electrodes which is just able to breakdown the gas between it $[1,2,3]$.

Atmospheric pressure discharge requires a very high voltage to be applied. Sufficiently high voltage of about $33 \mathrm{KV} / \mathrm{cm}$ is required to ionize the gas particles between electrodes $[2,3]$. These charged particles are responsible for the passage of current between 
electrodes. The nature of supplied voltage is immaterial but electrode gap and pressure of the gas plays vital role in the breakdown process. The discharge thus produced may be either of the types such as Townsend discharge, glow discharge and arc discharge which depends up on the current in the circuit $[2,3,4]$.

\section{EXPERIMENTAL}

A point-plane electrode system was fabricated as shown in Figure 1-2. High voltage $(11 \mathrm{KV}, 50 \mathrm{~Hz})$ was supplied to two electrodes that are set horizontally one above the other [2]. The upper electrode has some 30 identical pins of iron that are arranged parallel to each other on a copper plate and thus a comb shaped electrode is fabricated. The separation of each pin is $2 \mathrm{~mm}$. Every pin has its diameter $0.67 \mathrm{~mm}$ with discharging tip less than $0.1 \mathrm{~mm}$. The second electrode is a metal grid, which is also fabricated from iron, has length $7 \mathrm{~cm}$ and width $3 \mathrm{~cm}$. Each small square on the grid has area $1.52 \mathrm{~mm} \times 1.52$ $\mathrm{mm}$ and string of grid has diameter $0.2 \mathrm{~mm}$.

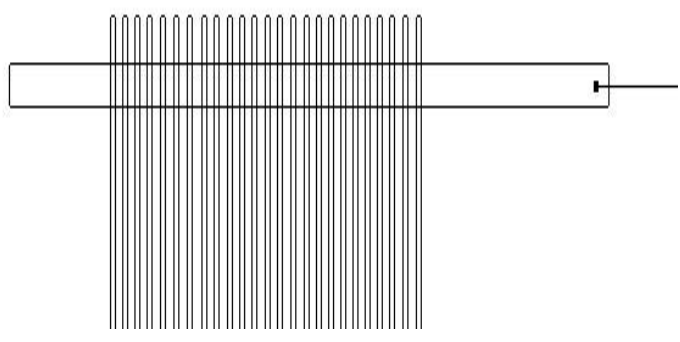

Fig1. Comb shaped electrode

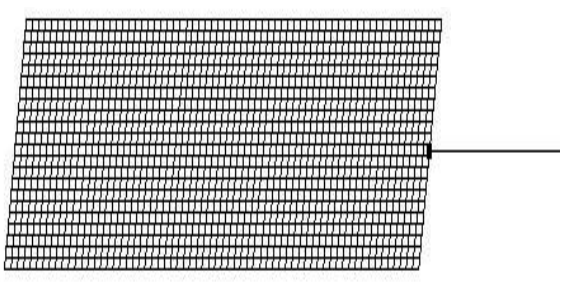

Fig2. Metal grid

The filamentary type discharge between electrodes was seen after the application of high voltage across them. These filamentary discharges turned in to homogenous bluish glow when lower electrode was covered by a thin polyethylene sheet.

To study the voltage and current characteristics of discharge, we used a high frequency digital oscilloscope. Voltage and current were measured from the resistive voltage divider circuit as shown in Figure 3.

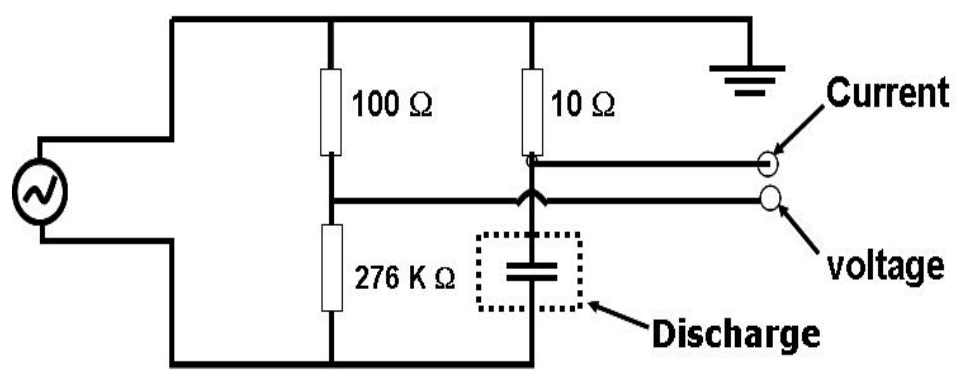

Fig3. Circuit diagram for measuring voltage and current

This circuit first lowers the high voltage to an acceptable range $(<100 \mathrm{~V})$ for oscilloscope and finds a multiplication factor for this low voltage. This low voltage is measured across the low resistance $100 \Omega$ connected in series with high resistance. The value of high resistance is $276 \mathrm{~K} \Omega$. The actual discharging high voltage can now be found out by 
multiplying the low voltage by this multiplication factor. The multiplication factor for this circuit is calculated to be 2.761. That means the voltage measured form oscilloscope should be multiplied by 2.761 to get the actual voltage in kilo-volts.

For the current measurement of discharge, a $10 \Omega$ resistor is used in the return path to the ground. Therefore, the actual discharge current will be equal to voltage across $10 \Omega$ resistor divided by 10 .

\section{RESULTS}

The current and voltage waveforms of the filamentary discharge were recorded with 1.8 mm discharge gap without any dielectric between electrodes. And the graphs for voltage and current were plotted by standard curve fitting method with the help of MicrocalOrigin data analyser software.

The oscillograph from the resistive voltage divider showed $1.8 \mathrm{~V}$. Therefore, the actual voltage for breakdown of gas was about $5 \mathrm{KV}$. Before breakdown, current was zero and at breakdown it sharply increases and became almost constant throughout the discharge phenomenon. The voltage across the $10 \Omega$ resistor was measured to be $500 \mathrm{mV}$, so the actual current would be $50 \mathrm{~mA}$. Thus, the power consumed by the discharge for breakdown was 248.49 watt. After breakdown, the voltage sharply decreases and becomes a constant value throughout the process. From oscillographs, the discharge voltage after breakdown was $0.925 \mathrm{~V}$. Therefore, the actual voltage after breakdown would be $2.554 K V$. Thus, the power consumed by reactor after breakdown was 127.7 watt.

When the lower electrode was covered by a thin dielectric material, the discharge turned into a homogenous bluish glow in air and voltage was slightly reduced. It was difficult to measure the change in voltage limited by the sensitivity of the oscilloscope. The current and voltage waveforms are depicted in Figure $4 \&$ Figure 5 respectively. The values for current is obtained from the values of voltage given in Figure 4 by dividing it by $10 \Omega$ resistance.

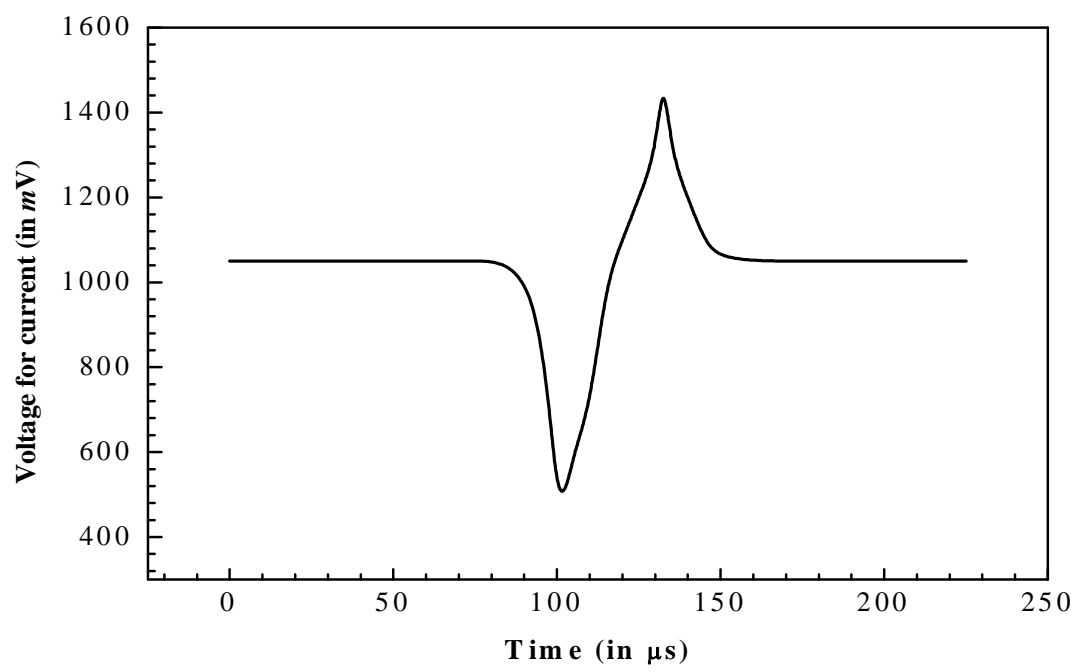

Fig4. Graph of voltage across $10 \mathrm{ohm}$ series resistor for current measurement 


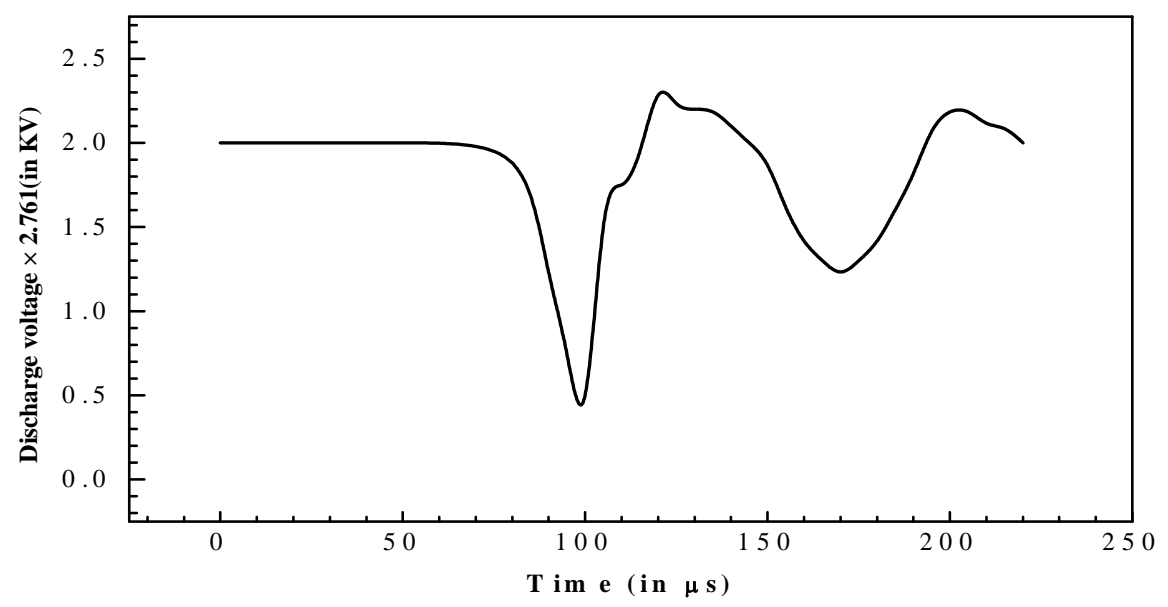

Fig 5. Graph of discharge voltage as a function of time

\section{CONCLUSIONS}

Gaseous discharge at atmospheric pressure in air was successfully generated in pointplane electrode system by applying high voltage across the electrodes. From the study of electrical characterization, it is clear that the discharge has low temperature because the thin polyethylene sheet was not damaged when inserted in the discharge region. Thus this discharge can be safely applied to processing of materials, purification of water, other bio-medical application and environmental application etc. Further work in this discharge system will be focused on the optical characterization by emission spectroscopy and determining the electron temperature by Boltzmann plot method.

\section{REFERENCES}

1. A. M. Howatson 1976. An Introduction to Gas Discharge. Second edition. Pergamon Press Ltd.

2. N. Jidenko, M. Petit and J.P. Borra 2006. Electrical Characterization of MicroDischarges Produced by Dielectric Barrire Discharge in Dry Air at Atmospheric Pressure. J. Phys. D: Appl. Phys. 39 281-293.

3. S. Okazaki, M. Kogoma, M. Ueharra, and Y. Kimura 1993. Appearance of Stable Glow Discharge in Air, Argon, Oxygen and Nitrogen at Atmospheric Pressure using a $50 \mathrm{~Hz}$ source. J. Phys. D: Appl. Phys., 26:889-892.

4. S. R. Kandel 2006. Production of Gaseous Discharge at Atmospheric Pressure and its Application in Treatment of Polyethylene Sheet. M. Sc. Thesis.

5. F.F. Chen 1594. Industrial Application of Low-Temperature Plasma Physics. University of California, Los Angeles, CA 90024.

6 U. Kogelschatz 1999. Discharge Physics, Plasma Chemistry and Industrial Applications. Annual Meeting Center for Plasma-Aided Manufacturing.Madison, Wisconsin, July 6-8. 
KATHMANDU UNIVERSITY JOURNAL OF SCIENCE, ENGINEERING AND TECHNOLOGY

VOL. I, No. V, SEPTEMBER 2008, pp 81-85.

7 D. P. Subedi 2002. Surface Modification of Polymers by Low Temperature Plasmas. Ph. D. Thesis, Department of Physical Electronics. Masaryk University, Kotlářská 2, 61137, Brno. Czech Republic. 